Document downloaded from:

http://hdl.handle.net/10251/147522

This paper must be cited as:

Min, R.; Marques, C.; Nielsen, K.; Bang, O.; Ortega Tamarit, B. (2018). Fast inscription of long period gratings in microstructured polymer optical fibers. IEEE Sensors Journal. 18(5):1919-1923. https://doi.org/10.1109/JSEN.2018.2791663

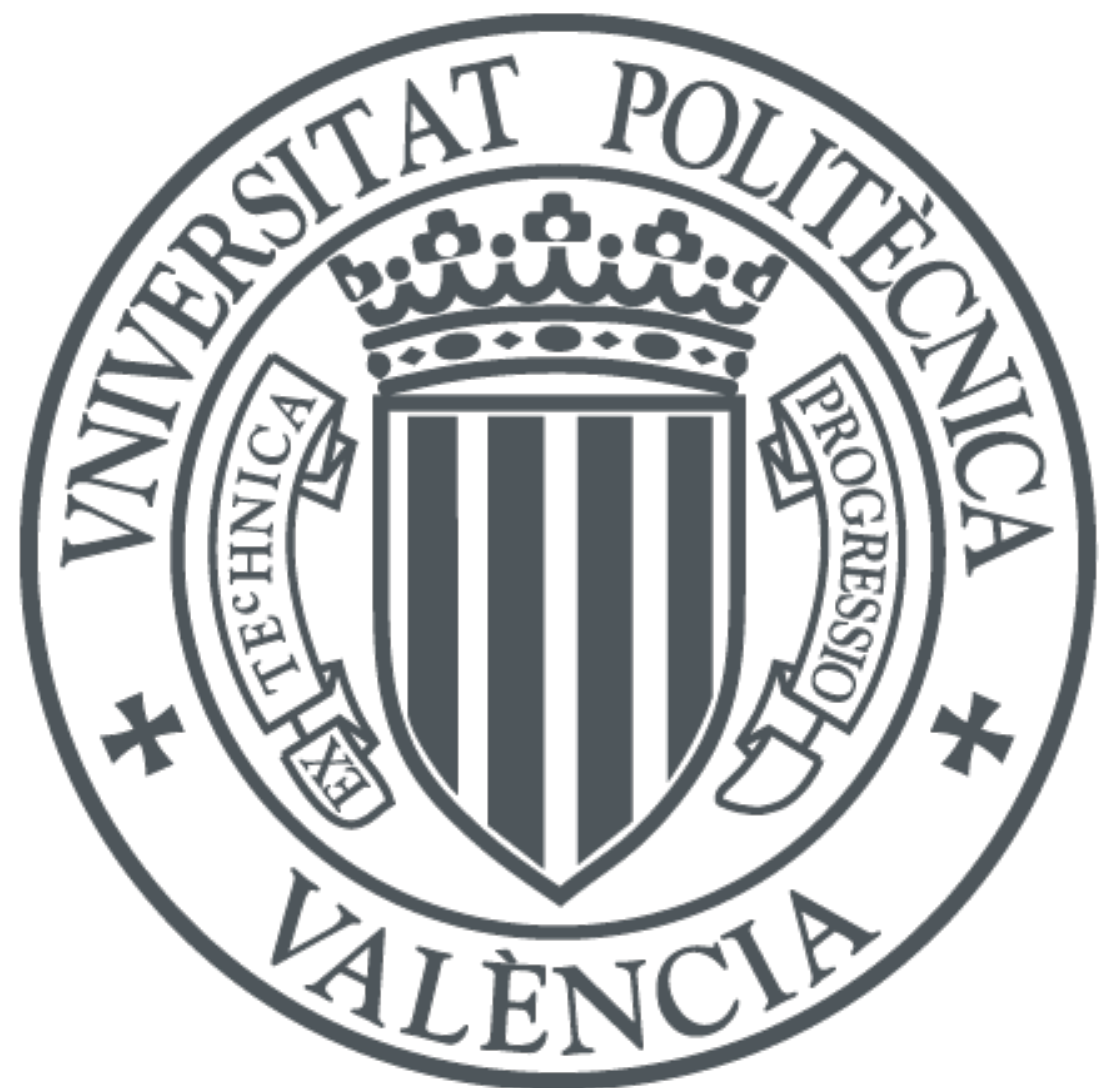

The final publication is available at

https://doi.org/10.1109/JSEN.2018.2791663

Copyright Institute of Electrical and Electronics Engineers

Additional Information

"(C) 2018 IEEE. Personal use of this material is permitted. Permission from IEEE must be obtained for all other uses, in any current or future media, including reprinting/republishing this material for advertisíng or promotional purposes, creating new collective works, for resale or redistribution to servers or lists, or reuse of any copyrighted component of this work in other works." 


\title{
Fast inscription of long period gratings in microstructured polymer optical fibers
}

\author{
Rui Min, Carlos Marques, K. Nielsen, Ole Bang, Beatriz Ortega, Member, IEEE
}

\begin{abstract}
We demonstrate $20 \mathrm{~dB}$ long period grating (LPG) fast inscription in microstructured polymer optical fibers (mPOFs) using a point-by-point technique obtaining an LPG total length of $25 \mathrm{~mm}$. Two $248 \mathrm{~nm}$ UV laser pulses of $15 \mathrm{~ns}$ duration have been employed for every inscription point, which means a time reduction by more than 21 times compared with the fastest inscription time already reported in literature. The device has been fabricated in a single-mode $\mathrm{mPOF}$ with a core that has been doped with benzyl dimethyl ketal (BDK) for photosensitivity enhancement. Moreover, we characterize the strain and temperature responses and the stability of the fabricated gratings response under different conditions in order to assess the viability for different applications.
\end{abstract}

Index Terms-Polymer optical fibers, fiber Bragg gratings, optical fiber devices, optical filters

\section{INTRODUCTION}

$\mathrm{D}$ URING the last two decades, long period fiber gratings (LPGs) have attracted significant attention due to their successful applications for both optical communications [1] and sensing [2]. The advantage of LPGs includes low insertion loss, ease of fabrication and low back reflection. Various LPGs based on silica fiber have been explored as WDM channel isolation filter [3], erbium-doped multi-wavelength fiber laser [4], highly sensitive refractometer [5] and sensor for different parameters such as strain [6], temperature [7], refractive index [8] and biochemical sensing [9,10]. Furthermore, properties of polymer optical fibers (POF) such as the large range of strain and small Young's modulus make LPGs even more attractive in these fibers. As a recent application, an all-plastic fiber-based pressure sensor based on LPG in microstructured POF (mPOF)

Manuscript received ......... The research leading to these results has received funding from the Fundação para a Ciência e Tecnologia (FCT)/MEC through national funds when applicable co-funded by FEDER - PT2020 partnership agreement under the projects UID/EEA/50008/2013 and this work has also been supported by the Research Excellence Award Programme GVA PROMETEO 2017/103 FUTURE MICROWAVE PHOTONIC TECHNOLOGIES AND APPLICATIONS and Fundamental Research Funds for the Heilongjiang Provincial Universities (KJCXZD201703). C. Marques also acknowledges the financial support from FCT through the fellowship SFRH/BPD/109458/2015. R. Min and B. Ortega are with the ITEAM Research Institute, Universitat Politècnica de València, Valencia, Spain (e-mail: rumi@doctor.upv.es, bortega@dcom.upv.es). C. Marques is with the Instituto de Telecomunicações and Physics Department \& I3N, Universidade de Aveiro, Portugal (e-mail: carlos.marques@ua.pt). K. Nielsen and O. Bang are with DTU Fotonik, Department of Photonics Engineering, Technical University of Denmark, SHUTE Sensing Solutions IVS, Centrifugevej 256, 2800 Kongens Lyngby, Denmark (e-mail: krini@ fotonik.dtu.dk, oban@fotonik.dtu.dk). has been explored [11]. However, the fabrication technology of LPGs in POF is still under research. Only a few papers mentioned the fabrication process of LPGs in mPOF [11-14]. The first LPG was fabricated in mPOF by mechanical deformation and heating [12]. UV photo inscription of LPG in $\mathrm{mPOF}$ was reported in 2010 with point-by-point technique [13]. Also, trans-4-stilbenmethanol was employed as external cladding dopant and demonstrated a reduced fabrication time down to six times compared to pure PMMA fibers with the same technique, which leads to reduce the coupling points as well as to shorten the irradiation time for each point $(42 \mathrm{~s}$ against $2 \mathrm{~min}$ ) [14]. In terms of minimizing fabrication times, which is a critical issue for commercial application, a $248 \mathrm{~nm}$ UV laser was used to inscribe a high quality fiber Bragg grating (FBG) in an undoped PMMA mPOF within less than $30 \mathrm{~s}$ [15]. Then, this laser type was employed to fabricate FBGs in benzyl dimethyl ketal (BDK) doped fiber with a single UV laser pulse [16] and it holds the minimum inscription time obtained until now. As demonstrated in previous work [16], BDK dopant acts as a photoinitiator which triggers a photo-polymerization process when irradiated with UV light and therefore, the photosensitivity of this fiber is increased.

To the best of our knowledge, in this letter we present the shortest inscription time for the LPG in an $\mathrm{mPOF}$, and we also investigate the temperature and strain sensitivities.

\section{LONG PERIOD FABRICATION GRATING FABRICATION}

A PMMA mPOF with 3 rings of holes and a BDK doped core was used to inscribe LPGs. The three ring cladding microstructure has a hole-to-pitch ratio of 0.47 with an average hole diameter of $1.74 \mathrm{um}$ and an average pitch of $3.70 \mathrm{um}$ [17], which makes it close to endlessly single-moded [18]. A cross-section image of the fiber is shown in Fig. 1. The fiber was annealed for 24 hours at 70 degrees before use in order to improve stability and quality of the inscribed gratings [19]. After cleaving it with a special room temperature cleaver [20] and polishing with sand paper, a long piece of fiber was connectorized and held onto the fabrication setup (see Fig. 2) under $0.1 \%$ strain, in order to ensure a good alignment between the fiber and UV beam. Several long pieces of fiber were connectorized to support the results of this work.

A Coherent Bragg Star Industrial-LN krypton fluoride (KrF) excimer laser system operating at $248 \mathrm{~nm}$ wavelength was employed for the LPG inscription. The laser pulse duration was $15 \mathrm{~ns}$ and the pulse energy was set at $2.95 \mathrm{~mJ}$. The laser beam 
profile was measured as a rectangular Tophat function of $6.0 \times 1.5 \mathrm{~mm}^{2}$ size and $2 \times 1 \mathrm{mrad}^{2}$ divergence. It was focused onto the fiber core utilizing a plano-convex cylindrical lens (Newport CSX200AR.10) with focal length of $20 \mathrm{~cm}$. The translation stage was employed to shift the laser beam with a precision of $10 \mu \mathrm{m}$ and a slit was employed to control the width of the beam where the point-by-point technique was employed to inscribe LPGs.

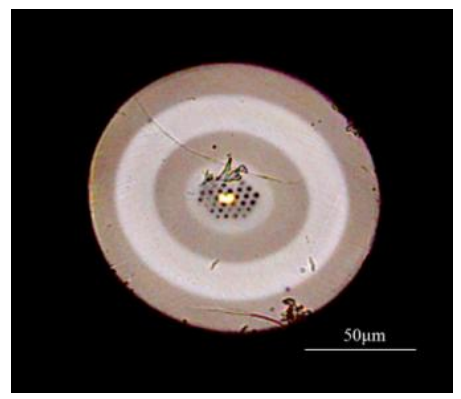

Fig. 1. Cross-section of the mPOF.

The grating transmission spectrum was monitored during fabrication by using a super luminescent diode (Superlum SLD-371-HP1) and an optical spectrum analyzer (Yokogawa AQ6373B) with $0.02 \mathrm{~nm}$ resolution bandwidth. According to the slit width, each point was $0.2 \mathrm{~mm}$ long, the beam was shifted $1 \mathrm{~mm}$ for inscribing every point and a total of 25 steps were completed, obtaining an LPG total length of $25 \mathrm{~mm}$. From these parameters the transmission values at the resonance of the LPG appear in the range of $800 \mathrm{~nm}$ to $900 \mathrm{~nm}$, as shown in previous works [13]. Different period values were tested however no significant results were obtained. Each inscription point was irradiated by two $15 \mathrm{~ns}$ pulses emitted by the UV laser at $1 \mathrm{~Hz}$ frequency repetition rate. Therefore, $2 \mathrm{~s}$ irradiation time is a significant reduction from the $42 \mathrm{~s}$ per point writing time reported in a previous work [14].

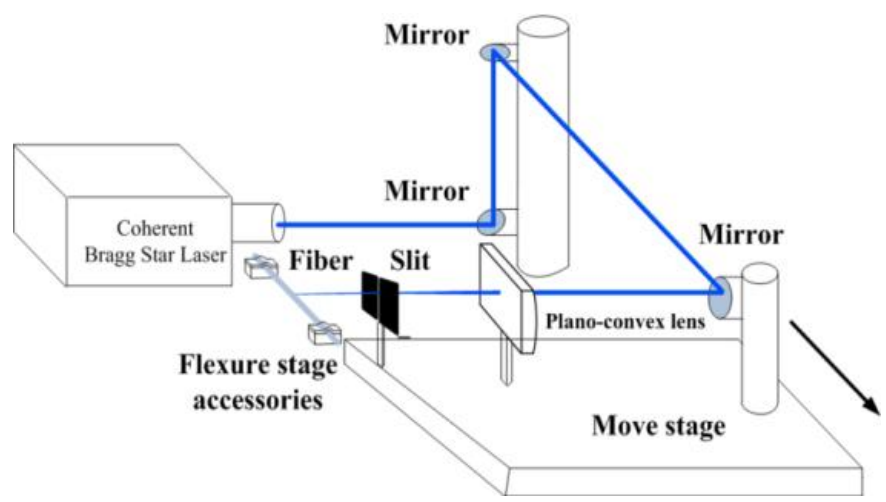

Fig. 2. Experimental setup for LPG inscription.

Fig. 3 shows the transmission of the LPG spectrum with only one irradiated pulse in each coupling point. The transmission of the grating was about $-3.0 \mathrm{~dB}$ at $870.05 \mathrm{~nm}$ just after fabrication. The results are presented as normalised transmission response respect to the output spectrum of the optical source. According with [16], just one $\mathrm{KrF}$ laser pulse with a duration of $15 \mathrm{~ns}$ introduces a refractive index change in the fiber core with enhanced photosensitivity by the BDK dopant. Also, it shall be noted that some experiments about single and multiple pulses were already explored for FBG inscription in [16]. In this way, both one and two pulses for each coupling point would lead to LPG fabrication, where the LPG inscription of $25 \mathrm{~mm}$ is achieved due to the BDK doped core of the fiber.

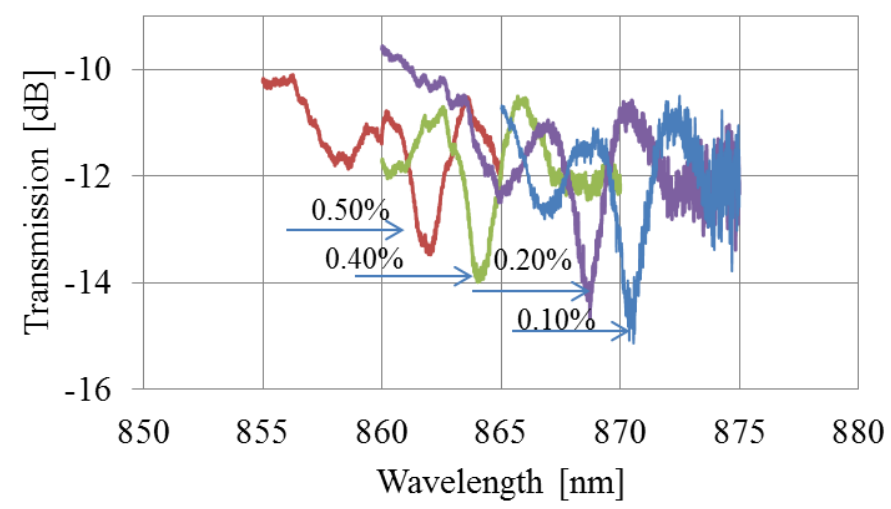

Fig. 3. Transmission of the LPG with one irradiated pulse per coupling point under different strain.

Another LPG was inscribed using two pulses for each coupling point in order to obtain a stronger grating. Fig. 4 shows the LPG transmission spectra just after fabrication when different levels of strain are applied. When the laser is stopped and the fiber is under $0.05 \%$ strain, the grating peak is centered at $869.5 \mathrm{~nm}$ and the transmission only drops $4.5 \mathrm{~dB}$, whereas the grating peak becomes more clear when it is further strained $(0.88 \%$ and $1.0 \%$ ), and intensity reaches around $9.0 \mathrm{~dB}$.

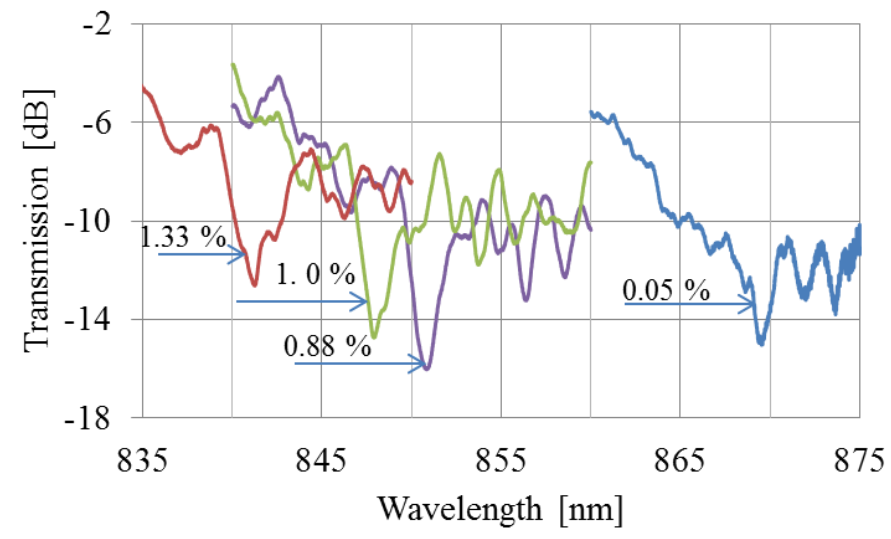

Fig. 4. Transmission of the LPG under different strain after inscription with two pulses for each coupling point.

However, after two weeks, the grating response shows a -20 $\mathrm{dB}$ peak centered at $866.9 \mathrm{~nm}$ wavelength when a $0.1 \%$ strain is applied, as shown in Fig 5. Such intensity growth after fabrication has been previously reported in FBG irradiated in annealed BDK doped PMMA fibers [16] and dye-doped 
PMMA polymer fibers [21]. After considerable number of experiments, we found that two UV pulses for each coupling point are a more reliable way to obtain LPGs with significant strength for sensing purposes.

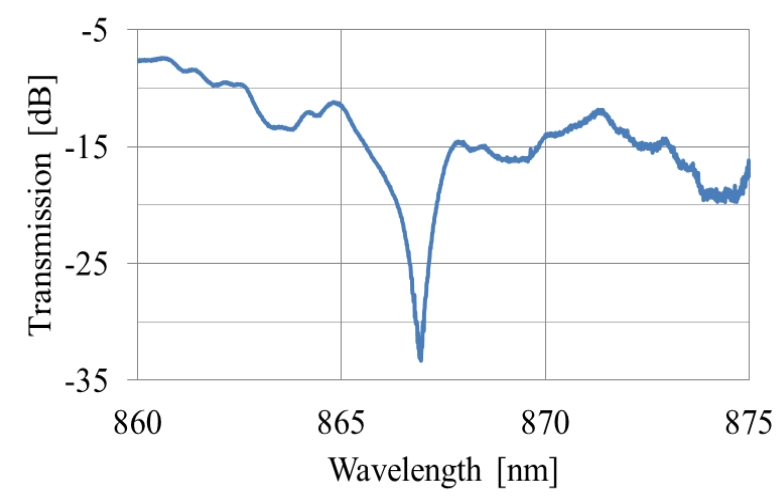

Fig. 5. Transmission of LPG with $0.1 \%$ strain two weeks after fabrication.

Transmission spectra were also collected after two weeks with different levels of strain measurement as shown in Fig. 6, and all cases show larger resonant dip than measured spectra just after fabrication, according to Fig. 4.

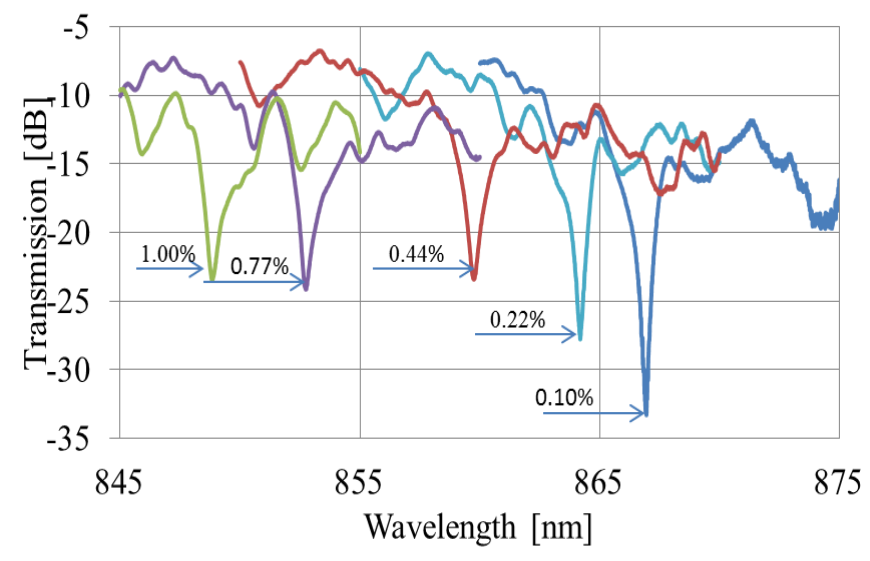

Fig. 6. LPG transmission responses under different strain after two weeks.

To achieve a detailed analysis of the LPG behavior under strain after fabrication (see Fig. 4) and two weeks after fabrication (see Fig. 6), the transmission values and the wavelength shift at the resonance of the LPG for different values of strain have been plotted in Fig. 7 for the sake of comparison. From Fig. 7 (a) we can observe a transmission depression of $20 \mathrm{~dB}$ when a $0.1 \%$ strain is applied and for $1 \%$ of strain the transmission depression decreases for $13 \mathrm{~dB}$. Fig. 7 (b) shows the peak wavelength variation for different strain levels and a sensitivity about $-2.21 \pm 0.05 \mathrm{~nm} / \mathrm{mstrain}$ was measured for the LPG after fabrication whereas, $-2.15 \pm 0.05 \mathrm{~nm} / \mathrm{mstrain}$ for the LPG two weeks after fabrication.

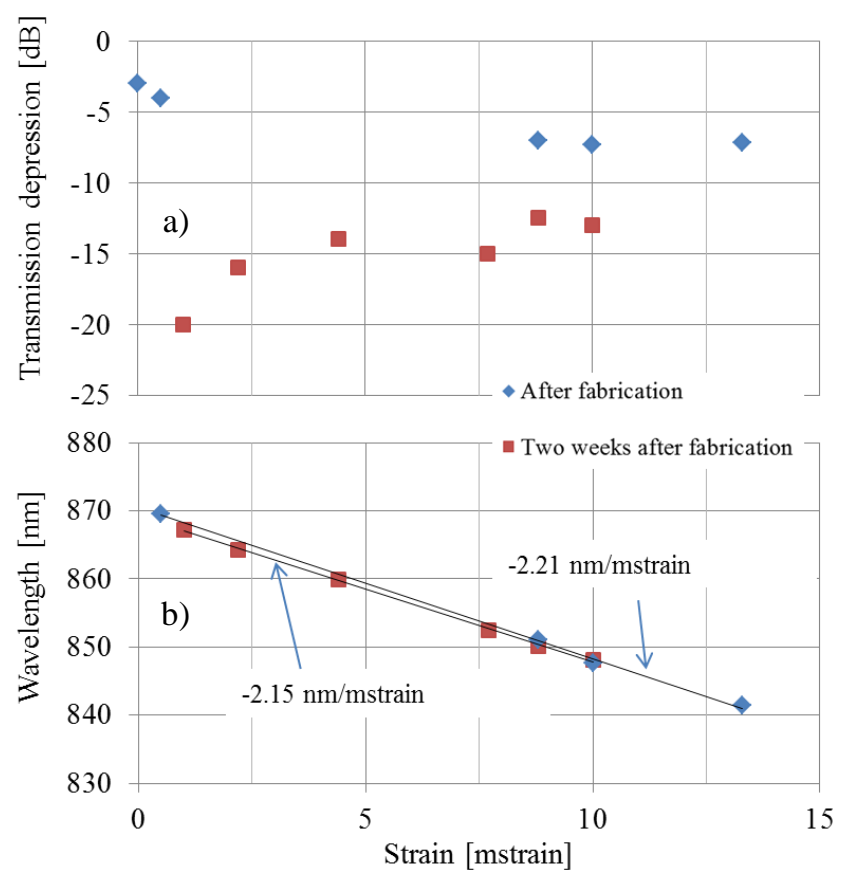

Fig. 7. LPG behavior under strain after fabrication and two weeks after fabrication. (a) Transmission depression of the grating. (b) Wavelength shift of the grating.

\section{STRAIN AND TEMPERATURE CHARACTERIZATION}

In the following, we measured the strain and temperature sensitivities of the fabricated LPGs in order to complete their characterization. To measure the former one, a $22 \mathrm{~cm}$ long connectorized fiber including an inscribed LPG was fixed with epoxy glue on the flexure stage accessories (Thorlabs THH001), and then fixed on the translation stage. The flexure stage fixing avoids sliding and also strain can be easily controlled by the translation stage. It shall be noted that we can use sharp blade to separate the fiber and the stage carefully for different experiments. The total fiber length between the glued points was about $18.1 \mathrm{~cm}$ and the length was changed by $20 \mu \mathrm{m}$ $(0.1105 \%$ strain $)$ in every measurement step. After 12 steps, a total $1.326 \%$ strain was applied and then, decreasing strain was applied by using the same steps.

Fig. 8 shows the peak wavelength variation against different levels of strain and sensitivity about $-2.30 \pm 0.05 \mathrm{~nm} / \mathrm{mstrain}$ was measured for increasing strain whereas, $-2.25 \pm 0.05$ $\mathrm{nm} / \mathrm{ms}$ train for decreasing strain due to polymer hysteresis. The measured sensitivity is slightly larger than presented in the literature, which ranges from -1.40 to $-1.44 \mathrm{~nm} / \mathrm{mstrain}$ for increasing strain and between -1.30 and $-1.40 \mathrm{~nm} / \mathrm{mstrain}$ for decreasing strain [22]. This phenomenon is probably due to fiber annealing for 24 hours at $70{ }^{\circ} \mathrm{C}$ as reported in [23] since the annealed fibers can produce an increase in the strain sensitivity.

We also studied the temperature response of the LPG inscribed which was fixed on the flexure stage and placed in the climatic chamber (Angelantoni Industrie CH340) with 50\% 
humidity under varying temperature values. The temperature was increased from $22{ }^{\circ} \mathrm{C}$ to $52{ }^{\circ} \mathrm{C}$ with steps of $5{ }^{\circ} \mathrm{C}$. Each temperature value was held during one hour to ensure stabilization.

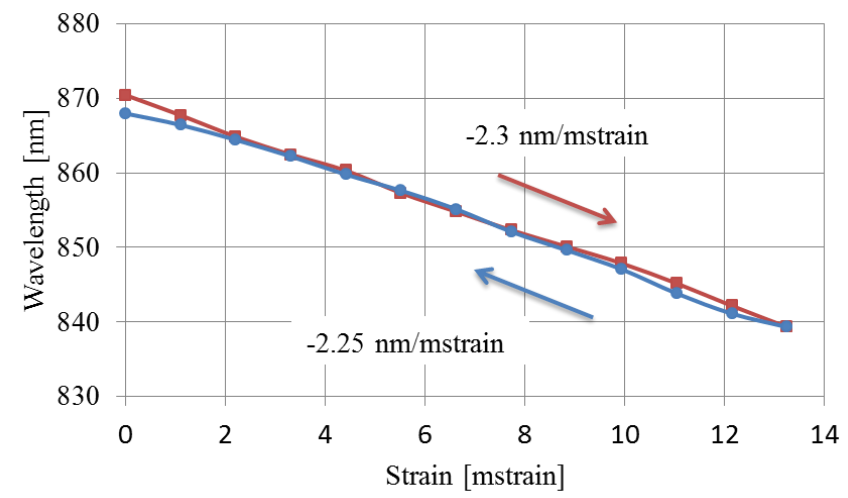

Fig. 8. Wavelength change induced by increasing and decreasing strain.

At the same time, we explored the stable behavior of the grating response during the characterization experiment. As depicted in Fig. 9, when the temperature is changed from $27^{\circ} \mathrm{C}$ to $32{ }^{\circ} \mathrm{C}$, the grating needs about 1 hour to become stable and before stability is reached, the grating shifts towards blue and then towards red wavelengths. However, when the temperature is increased from $47^{\circ} \mathrm{C}$ to $52^{\circ} \mathrm{C}$, the grating needs less than 10 minutes to become stable and central wavelength only shifts by a small amount.

The large range shift, observed for temperature change between $27^{\circ} \mathrm{C}$ and $42^{\circ} \mathrm{C}$ is probably due to the combined effect of humidity and temperature [24] since the water diffusion is a slow process. Oppositely, the grating becomes stable after a short time when the temperature is increased, as reported in [25], when the temperature is increased under 55\% relative humidity $(\mathrm{RH})$ conditions.

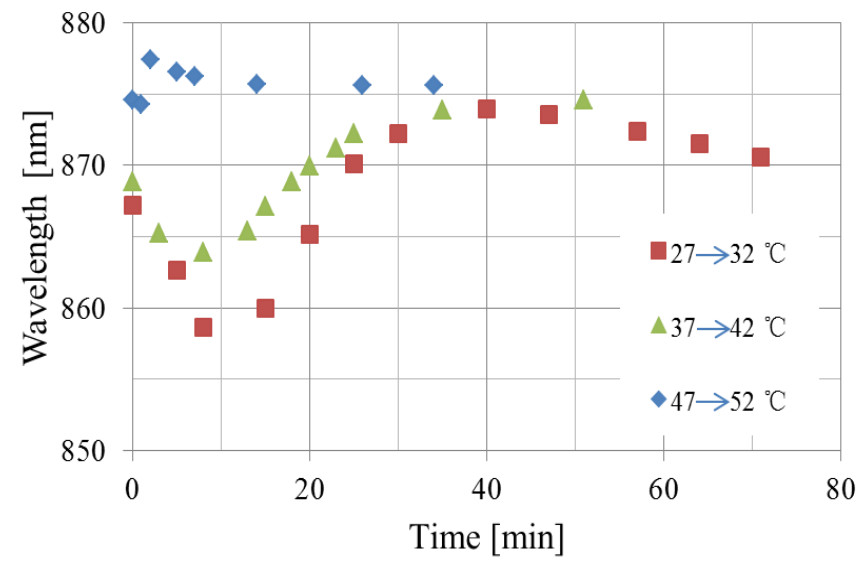

Fig. 9. Time evolution of the LPG central wavelength for different temperature variations.

Provided temperature is stabilized for each measurement, the obtained temperature sensitivity was $0.276 \pm 0.02 \mathrm{~nm} /{ }^{\circ} \mathrm{C}$, as shown in Fig. 10. The resonance wavelength of the grating shows a linear behavior with temperature. This behavior is different from previous results [22] where high nonlinearity and hysteresis was obtained when measurements were collected after $5 \mathrm{~min}$. The linear behavior is probably due to the larger measuring times employed in our experiment since we also found high nonlinearity in the grating behavior during the first 5 min due to the combination of temperature and humidity. Longer times ( $\sim 1$ hour) between each measurement allow to get a stable response and, consequently the wavelength shows a linear behavior with the temperature variation. Such behavior with temperature variation of LPG was also reported in $\mathrm{mPOF}$ with trans-4-stilbenmethanol as external cladding dopant [14]. However, the measured sensitivity is slightly larger than presented in the literature. From [14], in the linear part of the temperature characteristics the thermal sensitivity was roughly $0.035 \mathrm{~nm} /{ }^{\circ} \mathrm{C}$, which is almost one order less when compared with our result. It can be due to fiber drawing conditions, chemical composition of polymer and it is dependent of thermal history processing of each fiber.

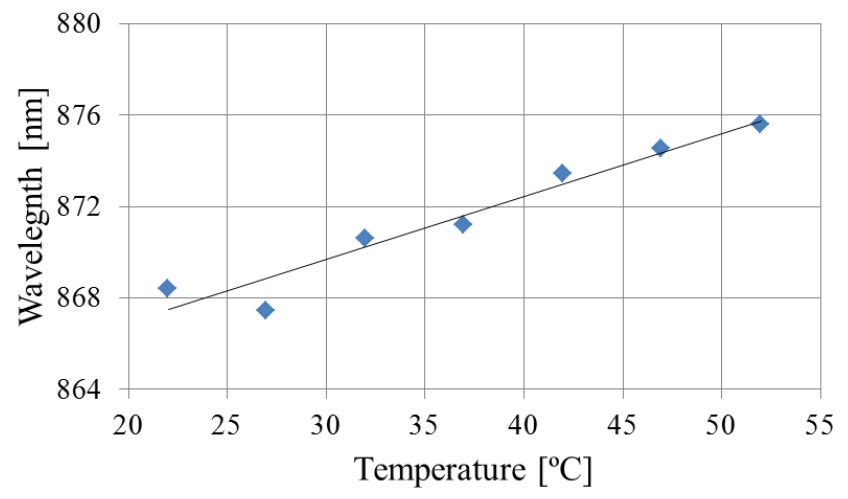

Fig. 10. Central wavelength dependence on the temperature.

Fig. 11 shows the transmission of the LPG after 50 days after fabrication, showing a good stability of the grating. The achieved strain sensitivity is $-1.97 \pm 0.05 \mathrm{~nm} / \mathrm{mstrain}$, slightly lower than before, which indicates the sensitivity decreases slowly with time.

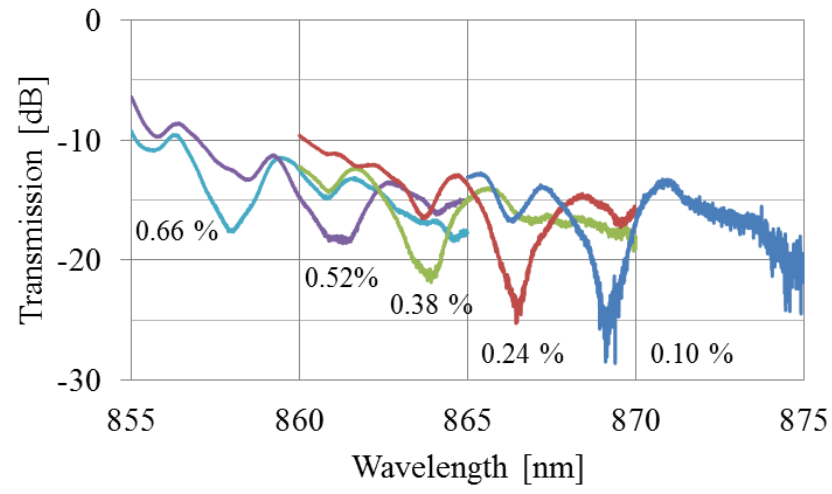

Fig. 11. LPG transmission behavior with strain (50 days after fabrication). 


\section{CONCLUSIONS}

To summarize, we demonstrated fast inscription of LPGs in polymer optical fibers using two $248 \mathrm{~nm}$ UV laser pulses per point by using point-by- point technique. The device has been fabricated in a single-mode mPOF which core has been doped with BDK for photosensitivity enhancement to UV radiation. The inscription time for each coupling point has been shortened by 21 times compared with the best one reported in the literature. The grating response shows a notch depth of $20 \mathrm{~dB}$ centered at $866.9 \mathrm{~nm}$ wavelength under $0.1 \%$ strain. The strain and thermal characterization of the LPG showed the strain and temperature sensitivity of $-2.3 \pm 0.05 \mathrm{~nm} / \mathrm{mstrain}$ and $0.276 \pm 0.02 \mathrm{~nm} /{ }^{\circ} \mathrm{C}$, respectively. Furthermore, we explored the stability of the grating response under different conditions to demonstrate its large potential for telecommunications and sensing applications.

\section{REFERENCES}

[1] A. M. Vengsarkar, P. J. Lemaire, J. B. Judkins, V. Bhatia, T. Erdogan and J. E. Sipe, "Long-period fiber gratings as band-rejection filters," $J$. Lightwave Technol., vol.14, no.1, pp. 58-65, 1996.

[2] S. W. James and R. P. Tatam, "Optical fibre long-period grating sensors: characteristics and application," Meas. Sci. Technol., vol.14, no.5, R49, 2003.

[3] X Liu, L Zhan, S Luo, Y Wang, "Individually switchable and widely tunable multiwavelength erbium-doped fiber laser based on cascaded mismatching long-period fiber gratings," J. Lightwave Technol., vol.29, no.21, pp. 3319-3326, 2011

[4] U. Tiwari, S. M. Tripathi, K. Thyagarajan, M. R. Shenoy, V. Mishra, S. Chander Jain, N. Singh, P. Kapur, "Tunable wavelength division multiplexing channel isolation filter based on dual chirped long-period fiber gratings," Opt. Lett, vol.36, no.19, pp.3747-3749, 2011.

[5] L. Rindorf, O. Bang, "Highly sensitive refractometer with a photonic-crystal-fiber long-period grating," Opt. Lett., vol.33, pp.563-565, 2008.

[6] X. Zhong, Y. Wang, J. Qu, C. Liao, S. Liu, J. Tang, Q. Wang, J. Zhao, K. .M Yang and Z. Y Li., "High-sensitivity strain sensor based on inflated long period fiber grating," Opt. Lett., vol. 39, no.18, pp.5463-5466, 2014.

[7] J. L. Li, W. G Zhang, S.C Gao, P.C Peng, X.L Xue, Z.Y Bai and H. Liang, "Long-period fiber grating cascaded to an $\mathrm{S}$ fiber taper for simultaneous measurement of temperature and refractive index," IEEE Photon. Technol. Lett., vol. 25, no.9, pp.888-891, 2013.

[8] J. F Ding, A. P Zhang, L.Y Shao, J.H Yan and S.L He, "Fiber-taper seeded long-period grating pair as a highly sensitive refractive-index sensor," IEEE Photon. Technol. Lett., vol.17, no.6, pp.1247-1249, 2005.

[9] L. Rindorf, J.B. Jensen, M. Dufva, P. E. Hoiby, L.H. Pedersen, O. Bang, "Photonic crystal fiber long-period gratings for biochemical sensing," Opt. Express. vol.14, pp. 8224-8231,2006.

[10] L. Rindorf, O. Bang, "Sensitivity of photonic crystal fiber grating sensors: biosensing, refractive index, strain, and temperature sensing," J. Opt. Soc. Am. B vol.25, no.3, pp.310-324, 2008.

[11] I. L Bundalo, R. Lwin, S. Leon-Saval, A. Argyros, (2016). "All-plastic fiber-based pressure sensor," Appl. Optics, vol. 55, no. 4, pp.811-816, 2016.

[12] M. P. Hiscocks, M. A. van Eijkelenborg, A. Argyros, M. C. J. Large, "Stable imprinting of long-period gratings in microstructured polymer optical fibre," Opt. Express, vol.14, no.11, pp. 4644-4649, 2006.

[13] D. Saez-Rodriguez, J. L. Cruz, I. Johnson, D. J Webb, M. C. J. Large, A. Argyros, "Water diffusion into UV inscripted long period grating in microstructured polymer fiber" IEEE Sens J., vol.10, no.7, pp.1169-1173,2010.

[14] D. Kowal, G. Statkiewicz-Barabach, P. Mergo and W. Urbanczyk "Microstructured polymer optical fiber for long period gratings fabrication using an ultraviolet laser beam," Opt. Lett, vol.39, no.8, pp.2242-2245, 2014.

[15] R. Oliveira, L. Bilro and R. Nogueira, "Bragg gratings in a few mode microstructured polymer optical fiber in less than 30 seconds," Opt. Express, vol.23, no.8, pp.10181-10187, 2015.

[16] A. Pospori, C.A.F. Marques, O. Bang, D.J Webb, and P. Andre, "Polymer optical fiber Bragg grating inscription with a single UV laser pulse," Opt. Express, vol.25,no.8, pp.9028-9038,2017.

[17] D. Saez-Rodriguez, K. Nielsen, H. K. Rasmussen, O. Bang and D.J. Webb, "Highly photosensitive polymethyl methacrylate microstructured polymer optical fiber with doped core,"Opt. Lett, vol.38, no.19, pp. 3769-3772, 2013.

[18] B. Kuhlmey, R. C. McPhedran, and C. M. de Sterke, "Modal cutoff in microstructured optical fibers," Opt. Lett., vol. 27, pp. 1684-1686, 2002.

[19] Yuan Wu, Alessio Stefani, Morten Bache, Torben Jacobsen, Bjarke Rose, Nicolai Herholdt-Rasmussen, Finn Kryger Nielsen et al., "Improved thermal and strain performance of annealed polymer optical fiber Bragg gratings," Opt. Communications, vol.284, no. 1 ,pp.176-182, 2011.

[20] D. Saez-Rodriguez, R. Min, B. Ortega, K. Nielsen and D.J. Webb, "Passive and Portable Polymer Optical Fiber Cleaver," IEEE Photon. Technol. Lett., vol.28, no.24, pp.2834-2837, 2016.

[21] H. B. Liu, H. Y. Liu, G.D Peng and P.L. Chu, "Novel growth behaviors of fiber Bragg gratings in polymer optical fiber under UV irradiation with low power," IEEE Photon. Technol. Lett., vol.16, no.1, pp.159-161, 2004.

[22] G. Statkiewicz-Barabach, D. Kowal, M.K. Szczurowski, P. Mergo and W. Urbanczyk, "Hydrostatic pressure and strain sensitivity of long period grating fabricated in polymer microstructured fiber," IEEE Photon. Technol. Lett., vol.25, pp.496-499, 2013.

[23] C. Marques, A. Pospori, G. Demirci, O. Çetinkaya, B. Gawdzik, P. Antunes, O. Bang, P. Mergo, P.S André, D. J. Webb, "Fast Bragg grating inscription in PMMA polymer optical fibres: impact of thermal pre-treatment of preforms," Sensors, Vol. 17, No. 4, pp. 891 - 891-8, 2017 ,

[24] W. Zhang, D. J. Webb, G.D Peng, "Investigation into time response of polymer fiber Bragg grating based humidity sensors," J. Lightwave Technol., vol.30, no. 8, pp. 1090-1096, 2012.

[25] W. Zhang, D.J. Webb, G.D Peng, "Enhancing the sensitivity of poly (methyl methacrylate) based optical fiber Bragg grating temperature sensors," Opt. Lett vol.40, pp. 4046-4049, 2015.

Rui Min received the B.S degree from Wuhan University of Technology, China, in 2010, and the M.S. degree from Harbin Engineering University, China, in 2015, now is currently pursuing the $\mathrm{Ph}$. D degree in Telecom at Universitat Politècnica de València, Spain. His research interests include optical fiber sensor, polymer fiber Bragg grating fabrication, microwave photonics.

Carlos Marques received the M.S. degree and the Ph.D. degree in Physics Engineering from the University of Aveiro, Portugal, in 2008 and 2013, respectively. From 2007 to March 2014, he was a Research Fellow in the Instituto de Telecomunicações, Aveiro, Portugal. Then, he was a Marie Curie Fellow in the Aston Institute of Photonic Technologies, Aston University, Birmingham, U.K until March 2016. Currently, he is a researcher in the Instituto de Telecomunicações and Physics Department \& I3N, University of Aveiro, Portugal. His main interests include optical fiber devices and fabrication of short/long period gratings for optical communications and sensing applications. He authored or co-authored more than 100 journal and conference technical papers with emphasis on optical communications and sensing applications.

Kristian Nielsen received the M.S. degree in physics and the Ph.D. degree from the Technical University of Denmark, Kongens Lyngby, Denmark, in 2008 and 2011, respectively. He is currently a Development Engineer with the Department of Photonics Engineering, Technical University of Denmark. His main interests include microstructured polymer optical fibers and their applications in sensing.

Ole Bang received the Master of Science degree in electrical engineering from the Technical University of Denmark in 1992 and the Doctor of Philosophy degree in nonlinear physics from the Technical University of Denmark, in 1993.From 1993 to 1995, he was a Postdoctoral Fellow the Laboratoire de Physique, Ecole Normale Supérieure de Lyon, France, where he worked on discrete physical models of the nonlinear dynamics of biomolecules. From 1995 to 1999, he was a Research Fellow at the Optical Sciences Centre, 
Australian National University, Canberra, Australia, where he worked theoretically on nonlinear optics, in particular on solitons and modulation instability in materials with a quadratic nonlinearity and quasi-phase-matching gratings. Since 1999, he has been an Associate Professor with the Technical University of Denmark, first at the Department of Informatics and Mathematical Modelling and, since 2003, with DTU Fotonik, Department of Photonics Engineering.His current research interests include microstructured optical fibers (MOFs) in silica and polymer, fiber-optical sensors for sensing biomolecules, stress, sound, and refractive index. He is also working on fabricating Bragg gratings, long-period gratings, and couplers in MOFs. Another main interest is super-Continuum generation and nonlinear fiber-optics in MOFs, as well as general nonlinear optics in nonlocal materials.
Beatriz Ortega received the M.Sc. degree in Physics in 1995 from the Universidad de Valencia, and the Ph.D. in Telecommunications Engineering in 1999 from the Universidad Politécnica de Valencia. Currently working as full professor at the Departamento de Comunicaciones from the Universitat Politècnica de València and collaborating as a group leader in the Photonics Research Labs in the Institute of Telecommunications and Multimedia Applications. She has published more than 200 papers and conference contributions in fibre Bragg gratings, microwave photonics and optical networks. She has got several patents and is also a co-founder of EPHOOX Company. She has participated in a large number of European Networks of Excellence and R\&D projects and other national ones. Her main research is currently focused on optical devices, optical networks and microwave photonic systems and applications. 